\title{
Forward-Inverse Adaptive Techniques for Reservoir Characterization and Simulation. Theory and Applications
}

S.K. Doss, S. Ezzedine, R.J. Gelinas, J.P. Ziagos, A. Chawathe

This paper was presented at the 2001 Society of Petroleum Engineers Annual Technical Conference and Exhibition, New Orleans, LA,

\section{September 1, 2001}




\section{DISCLAIMER}

This document was prepared as an account of work sponsored by an agency of the United States Government. Neither the United States Government nor the University of California nor any of their employees, makes any warranty, express or implied, or assumes any legal liability or responsibility for the accuracy, completeness, or usefulness of any information, apparatus, product, or process disclosed, or represents that its use would not infringe privately owned rights. Reference herein to any specific commercial product, process, or service by trade name, trademark, manufacturer, or otherwise, does not necessarily constitute or imply its endorsement, recommendation, or favoring by the United States Government or the University of California. The views and opinions of authors expressed herein do not necessarily state or reflect those of the United States Government or the University of California, and shall not be used for advertising or product endorsement purposes.

This is a preprint of a paper intended for publication in a journal or proceedings. Since changes may be made before publication, this preprint is made available with the understanding that it will not be cited or reproduced without the permission of the author.

This report has been reproduced directly from the best available copy.

Available electronically at http://www.doc.gov/bridge

Available for a processing fee to U.S. Department of Energy

And its contractors in paper from

U.S. Department of Energy

Office of Scientific and Technical Information

P.O. Box 62

Oak Ridge, TN 37831-0062

Telephone: (865) 576-8401

Facsimile: (865) 576-5728

E-mail: reports@adonis.osti.gov

Available for the sale to the public from

U.S. Department of Commerce

National Technical Information Service

5285 Port Royal Road

Springfield, VA 22161

Telephone: (800) 553-6847

Facsimile: (703) 605-6900

E-mail: orders@ntis.fedworld.gov

Online ordering: http:/ / www.ntis.gov/ordering.htm

\section{OR}

Lawrence Livermore National Laboratory

Technical Information Department's Digital Library

http: / / www.llnl.gov/tid/Library.html 


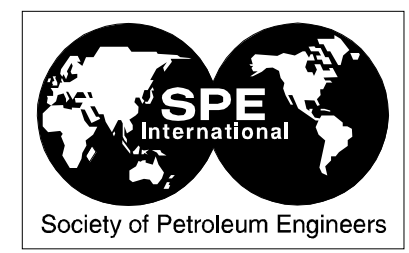

\section{Forward-Inverse Adaptive Techniques for Reservoir Characterization and Simulation. Theory and Applications

\author{
S.K. Doss, Lawrence Livermore National Laboratory, S. Ezzedine, Weiss Associates, R.J. Gelinas, J.P. Ziagos, LLNL, \\ and A. Chawathé, SPE, Chevron Petroleum Tech. Co
}

This paper was prepared for presentation at the 2001 SPE Annual Technical Conference and Exhibition held in New Orleans, Louisiana, 30 September-3 October 2001.

This paper was selected for presentation by an SPE Program Committee following review of information contained in an abstract submitted by the author(s). Contents of the paper, as presented, have not been reviewed by the Society of Petroleum Engineers and are subject to correction by the author(s). The material, as presented, does not necessarily reflect any position of the Society of Petroleum Engineers, its officers, or members. Papers presented at SPE meetings are subject to publication review by Editorial Committees of the Society of Petroleum Engineers. Electronic reproduction, distribution, or storage of any part of this paper Petroleum Engineers. Electronic reproduction, distribution, or storage of any part of this paper
for commercial purposes without the written consent of the Society of Petroleum Engineers is prohibited. Permission to reproduce in print is restricted to an abstract of not more than 300 words; illustrations may not be copied. The abstract must contain conspicuous acknowledgment of where and by whom the paper was presented. Write Librarian, SPE, P.O. Box 833836, Richardson, TX 75083-3836, U.S.A., fax 01-972-952-9435.

\begin{abstract}
A novel approach called Forward-Inverse Adaptive Techniques (FIAT) for reservoir characterization is developed and applied to three representative exploration cases. Inverse modeling refers to the determination of the entire reservoir permeability under steady state single-phase flow regime, given only field permeability, pressure and production well measurements. FIAT solves the forward and inverse partial differential equations (PDEs) simultaneously by adding a regularization term and filtering pressure gradients. An implicit adaptive-grid, Galerkin, numerical scheme is used to numerically solve the set of PDEs subject to pressure and permeability boundary conditions. Three examples are presented. Results from all three cases demonstrate attainable and reasonably accurate solutions and, more importantly, provide insights into the consequences of data undersampling.
\end{abstract}

\section{Introduction}

The problem of reservoir characterization has attracted a remarkable research effort, particularly over the last 20 years. Research in the area of site characterization has focused mainly on development of inverse algorithms ${ }^{1,2,3}$. While our understanding of the problem has improved, the problem is generally considered as yet unsolved with no fully proven technique, there are clear ideas of where the weak points are and what remedies might be.

It is widely recognized now that natural heterogeneity and the large spatial variability of the permeability and porosity predominantly control the flow field and hence forecasting of reservoir performance, optimization of management strategies, placement of infill and production wells, and other related practical reservoir engineering decisions. Oil reservoirs are usually undersampled relative to the spatial variability of the porous media properties that may also vary largely in magnitude over very short distances. As a result, there is not enough point measurements to provide a comprehensive and detailed characterization. Clearly, however, scant knowledge of essential reservoir parameters and operational data does not minimize the potential such limited information can provide if utilized consistently and maximally.

Most literature on the subject dealing with the solution of the inverse problem appears to favor the optimal control theory approach, and the great majority of that work reduces, essentially, to a least-square minimization technique on the pressure variable constrained by the time-dependent production. Fewer works have gone further to incorporate additional information such as geophysical measurements ${ }^{4}$. Still other researchers solve the mass balance equation for $\mathrm{K}$ given $\mathrm{P}$, commonly called the inverse PDE problem, and ensuing methods are called "direct" approaches". For some pressure conditions and geological reservoir settings, decoupling the forward model from the inverse one, in the direct approach is probably acceptable. But for heterogeneous geological settings and for reservoirs under extreme stresses, the decoupling assumption usually does not hold and the direct inverse solution requires accurate pressure gradient determinations which are extremely difficult to obtain because measurements are sparsely available and at disparate scales.

It is pertinent to cite the work of $\mathrm{Zhu}^{6}$ in which a history matching procedure was suggested through cyclic solution of forward and inverse system of equations. Zhu's approach resembles the approach presented here, though the details are markedly different. The iterative scheme in Zhu's approach starts by solving the forward problem in the pressure assuming a known permeability. Then, a new system of algebraic equations in $\mathrm{K}$ is constructed from the forward system by switching the role of $\mathrm{P}$ and $\mathrm{K}$ ( $\mathrm{K}$ becomes the unknown whereas $\mathrm{P}$ is given). This new system represents then the inverse portion in each cycle. Any additional constraints on $\mathrm{K}$ are invoked in this portion through the modification of the coefficient matrix. After solving for $\mathrm{K}$, the cycle is repeated until the calculated pressure is satisfactorily matched with the measured one. Notice that the construction of the inverse system in this approach is in itself not new; for instance Sun ${ }^{5}$ describes a similar procedure to extract a set of inverse equations from forward ones; what is interesting here is its utilization alongside the forward system. In contrast to Zhu's approach, the inverse portion in this work derives its Galerkin equations directly from the inverse PDE (in lieu of switching the roles of $\mathrm{P}$ and $\mathrm{K}$ in the Galerkin equations for the forward $\mathrm{PDE})^{7}$. In essence, the solution to the inverse PDE is implicitly coupled to the forward one through a nearly degenerate set of 
partial differential equations. Although, this coupling usually creates a highly nonlinear and numerically challenging system of partial differential equations; on the other hand, it ensures that the solution of the inverse problem is, at least, always consistent with the forward solution everywhere in the reservoir. The present paper is composed of 5 sections. Section 2 states the inverse problem. Section 3 presents the forward inverse algorithm and its numerical solution. Section 4 covers the proof-of-concept examples and applications. All findings are summarized in Section 5.

\section{Statement of the problem}

To emphasize basic concepts without loss of generality, the steady-state single-phase flow in a confined reservoir is described by the following equation:

$$
\nabla \cdot[\mathrm{K}(\mathbf{x}) \nabla \mathrm{P}(\mathbf{x})]=0 .
$$

$\mathrm{K}$ is the permeability of the reservoir. $\mathrm{P}$ is the reservoir pressure. $\mathbf{x}$ denotes a point in space, and boldface denotes vectors. A reservoir is characterized by borehole measurements of permeability, pressure, and production rate. These measurements are dispersed in the domain and they are measurement-scale depend. The domain is denoted by $\Omega$, and its boundaries by $\partial \Omega$. The thickness of the aquifer is assumed known from measurements, as are pumping and injection rates. Equation (1) is called the forward equation or the pressure equation. The forward problem assumes that $K(\mathbf{x})$ is known throughout $\Omega$ and $\mathrm{P}(\mathbf{x})$ is the only unknown to be determined, given a set of boundary conditions of first and/or second type. Equation (1) is elliptic and its numerical solution is easy to attain. In reality, however, $\mathrm{K}(\mathbf{x})$ is only known at a limited number of sparse measurement locations in $\Omega$. Other measurements such as pressure and injection and/or production rates are also available at different or same $\mathrm{K}$ measurement locations. Inverting for the permeability $\mathrm{K}(\mathbf{x})$, equation (1) becomes hyperbolic and presents serious numerical challenges. In this case (1) is referred to as the inverse equation or the K-equation. The inverse problem is an improperly or ill-posed problem ${ }^{5}$ and some modifications need to be considered to assure convergence to a stable solution of (1). Mathematically speaking, the inverse problem is a Cauchy one. It has been shown that the inverse problem has a unique stable solution when $\mathrm{P}(\mathrm{x})$ is known in the entire domain and a "K-boundary" condition is given along a Cauchy line, $\ell$, crossing every streamline in $\Omega$. Cauchy solutions did not attract the attention of reservoir practioners because K-data along $\ell$ are hardly ever available in practice. Theoretical advances have been made $\mathrm{e}^{8,9,10}$ but without successful application. Reservoir practitioners have scattered their attention toward direct or indirect techniques such as gradient techniques, minimization or optimization techniques, and more recently stochastic and geostatistical techniques. Regrettably, the inverse problem was always considered independent from the forward one, but constrained by heuristic and interpolation techniques that led to inconsistencies between the forward and the inverse problem solutions. Consequently, our goal is twofold. First, to assure consistency between both problems, we aim to simultaneously solve the inverse and forward problems, through an implicit scheme using an adaptive Galerkin FE technique. Second, we intend to show that by moving the Cauchy "lines" to the production/injection wells and by unspecifying making the Kboundary on $\partial \Omega$ a "non-specified" boundary, we can reach a more appreciable physical solution to the problem.

\section{Forward-Inverse Adaptive Algorithm}

Let $\left\{{ }^{\mathrm{P}} \mathbf{X}\right\}, \quad\left\{{ }^{\mathrm{K}} \mathbf{X}\right\}, \quad\left\{{ }^{\mathrm{Q}} \mathbf{X}\right\}$ denote finite sets of Cartesian coordinates at which measurements of pressure, permeability, and production are collected, respectively. The 3D Cartesian coordinates are denoted by $\left\{\left(\mathrm{j}_{1, i}^{\mathrm{j}}, \mathrm{x}_{2, \mathrm{i}}, \mathrm{x}_{3, \mathrm{i}}\right), \mathrm{i}=1,2, \ldots, \mathrm{n}^{\mathrm{j}}\right.$, and $\mathrm{j}=\mathrm{P}, \mathrm{K}, \mathrm{Q}\} . \mathrm{n}^{\mathrm{j}}$ are the number of measurements of $\mathrm{j}$-type. Without loss of generality, we treat the case where all $n^{j}$ are equal. This 'fixed point set' becomes part of the nodal assembly in a finite elements solution of the governing PDEs.

Forward Problem. The forward problem consists of solving (1) subject to given P measurements at $\left\{{ }^{\mathrm{P}} \mathbf{X}\right\}$ and pressure and flux boundary conditions on $\partial \Omega$. The outcome of this step is $\mathrm{P}$ and thus the estimation of pressure gradients $\nabla \mathrm{P}(\mathbf{x})$ for the inverse problem step. Sparsely measured pressure data (as well as sparse permeability data) present both the major problem and the reason for permeability parameter estimation. Because of the lack of complete knowledge of $\mathrm{P}$ everywhere in $\Omega$, it follows, that pressure gradients are not defined in all of $\Omega$; and thus a PDE does not exist for K. An immediate way to proceed is to find an interpolating surface that passes through all of the pressure datum points.

Spatial Filtering. Because a vast number of interpolating surfaces often exist, the inverse solution, if found, will often yield to unphysical permeability solution unless further constraints are imposed. The cause of this difficulty is readily explained: most trial interpolating surfaces that pass through the measured pressure datum points form relative maxima or minima 'spikes' on the set $\left\{{ }^{\mathrm{P}} \mathbf{X}\right\}$, thereby violating the maximum-principle for elliptic differential equations ${ }^{11}$. The interpolating surfaces must therefore be selected judiciouslywhich is the starting point for the developments in this work. Because stochastic methods need a large and sufficient number of data for statistical inference, geostatistical characterization and interpolation of $\mathrm{P}$ away from the data locations, one can resort to a classic spatial filtering technique of the gradients. Indeed, much of the important information content from pressure measurements, no matter how sparse or devoid of high spatial frequency information they may be, resides in the gradients and Laplacians of the pressure. The objective of this step is to extract as much information as possible by performing spatial filtering that is commensurate with the spatial intervals between the measured data ${ }^{12}$. Such filtering is also required to produce a smoothed map pressure that, effectively, satisfies the maximum principle for elliptic differential equations. The clamping imposed on $\mathrm{P}$ is therefore relaxed at the measurement points by solving the following PDE for $\nabla \mathrm{P}$ on $\Omega$ :

$$
\Delta\left[\nabla \mathrm{P}_{\mathrm{S}}(\mathbf{x})\right]+\alpha\left(\nabla \mathrm{P}(\mathbf{x})-\nabla \mathrm{P}_{\mathrm{S}}(\mathbf{x})\right)=0 \text { in } \Omega
$$

The Laplacian operation in the first term of equation (2) is a band-pass filter function ${ }^{13}$. It does not have a sharp cut-off at any spatial frequency; so some latitude can be exercised in Laplacian smoothing with this factor in mind. The second term in equation (2) can be viewed as a penalty function that controls the amount of smoothing of $\mathrm{P}$ through proper choices 
of the parameter $\alpha$. Clearly, very large $\alpha$ yields a $P_{S}$ that hardly differs from $\mathrm{P}$; that is, very little smoothing is done. And vice versa, as $\alpha$ approaches zero, $P_{S}$ approaches a harmonic solution in $\Omega$. But too much smoothing is obviously undesirable when it loses (by aliasing) significant amounts of information. The rationale for this step is to find the major regional driven gradients. The solution $\mathrm{P}_{\mathrm{S}}$ is then used to approximate $\nabla \mathrm{P}(\mathbf{x}) \approx \nabla \mathrm{P}_{\mathrm{S}}(\mathbf{x})$ that is needed for the inverse problem step.

Inverse Problem. $\nabla \mathrm{P}_{\mathrm{S}}(\mathbf{x})$ is the desired smoothed pressure gradient, relative to less-smooth gradients $\nabla \mathrm{P}(\mathbf{x})$, the following inverse problem PDE is introduced and solved for $\mathrm{K}(\mathbf{x})$ in $\Omega$ :

$$
\nabla \cdot\left[\mathrm{K}(\mathbf{x}) \nabla \mathrm{P}_{\mathrm{S}}(\mathbf{x})\right]+\varepsilon \Delta \mathrm{K}(\mathbf{x})=0 \text { in } \Omega
$$

This modified equation (3), through the addition of the regularizing term $\varepsilon \Delta \mathrm{K}$, is in principle an elliptic equation. But in practice it is essentially a hyperbolic PDE. The solution to equation (3) can then be completely determined, provided that $\mathrm{K}$ is given along some non-characteristic curve, $\ell$, within $\Omega$ and such that the continuum of characteristics emanating from $\ell$ spans the entire domain $\Omega$. For brevity, an $\ell$-curve that satisfies these conditions will be referred to as an admissible $\ell$ curve. If no admissible $\ell$-curve exists for the entire domain $\Omega$, then one must partition $\Omega$ into two or more subdomains, each of which possesses its own admissible $\ell$-curve. No further specification of $\mathrm{K}$ along the remainder of $\partial \Omega$ is required. Such a 'non specified' boundary condition is exacted by recycling integrands in all boundary integrals that are produced from integration by parts in the numerical solution process ${ }^{14}$. That is, whatever expression appears in a boundary integral is reused, as is, in forming the matrix of the Galerkin approximation equations.

Forward-Inverse Adaptive Techniques (FIAT). To assure consistency between the forward and the inverse problem, we propose to implicitly and simultaneously solve equations (1)(3) using an adaptive Galerkin finite element method. The system of PDEs is highly nonlinear; in addition, we are required to "stage" the regularization term, $\varepsilon$, to guarantee a stable numerical scheme of the system of PDEs.

Boundary Conditions. In the present study we distinguish two classes of boundary conditions (BCs):

1) Outer-domain $\mathrm{BCs}$ are dictated mainly by the geological reservoir delineations. For the pressure equation (1), such $\mathrm{BCs}$ are specified pressures (i.e., value $(\mathrm{P}))$ or fluxes (i.e., natural $(\mathrm{P}))$. For the $\mathrm{K}$ equation (2), similar BCs are of unspecified (open) BC-type, or if the permeability is known over a portion of $\partial \Omega$, a specified $\mathrm{BC}$ is straightforward (i.e., value $(\mathrm{K}))$. It is worthwhile to note that because of the reciprocity between $\mathrm{K}$ and $\nabla \mathrm{P}$, a null flux $\mathrm{BC}$ leads to a zero "K-flux" (i.e., $\operatorname{natural}(\mathrm{K})=0)$.

2) Inner-domain BCs are dictated by $P, K$, and $Q$ field measurements. $\mathrm{P}$ and $\mathrm{K}$ measurements are scale dependent. Also, whenever the triplet $\{\mathrm{P}, \mathrm{K}, \mathrm{Q}\}$ are given at the same location they must satisfy Darcy's Law. Permeability and pressure are not point-function but set-function measurements and their measurement support differs from one measurement to another ${ }^{15}$. Because fluxes integrate streamlines emanating from as far as the reservoir outer boundaries, they are considered regional measurements. Therefore, flux measurement support is larger than $\mathrm{P}$ and $\mathrm{K}$ measurement supports. Often the extent of $\mathrm{K}$ heterogeneity is larger than pressure monitoring or production/injection well diameters. If we denote by $\omega_{\mathrm{P}}, \omega_{\mathrm{K}}$, and $\omega_{\mathrm{Q}}$, the measurement support of $\mathrm{P}, \mathrm{K}$, and $\mathrm{Q}$, respectively, we conjecture that:

$\omega_{\mathrm{P}} \subseteq \omega_{\mathrm{K}} \subseteq \omega_{\mathrm{Q}} \subset \Omega$.

Based on the above support hierarchy the triplet $\{P, K, Q\}$ is assigned around the data $\left\{{ }^{\mathrm{P}} \mathrm{X}\right\},\left\{{ }^{\mathrm{K}} \mathrm{X}\right\}$, $\left\{{ }^{\mathrm{Q}} \mathrm{X}\right\}$ point-location center. Consequently, three fictitious radii, $\rho_{i}$, are introduced, and each corresponds to $\omega_{i}$. The $\mathrm{P}$ and $\mathrm{K}$ measurements are specified-value $\mathrm{BCs}$ assigned on the perimeter, or the vertical lateral surface of $\omega_{\mathrm{Q}}$ in $2 \mathrm{D}$ or $3 \mathrm{D}$ cases, respectively. The flux measurements, however, are specified-flux BCs. Whenever $\{\mathrm{P}, \mathrm{K}, \mathrm{Q}\}$ are given at any point it results in hyper-point data. While $\mathrm{P}$, and $\mathrm{K}$ are used as BCs around the well, fluxes are used as constraints in FIAT. At each production or injection well the following condition has to be satisfied:

$\oint_{2 \pi \rho_{Q_{i}}} K_{i} \frac{\partial P}{\partial r} r d \theta=Q_{i}, \quad i=1, \cdots, n^{Q}$.

Equation (5) is used to constrain the $\mathrm{P}$ and $\mathrm{K}$ solutions in the neighborhood of the well. FIAT easily incorporates constraints using Lagrangian multiplier technique ${ }^{16}$. Because $\mathrm{P}, \mathrm{K}$, and $\mathrm{Q}$ can be measured at the same well and they embed different measurement supports, they have to obey Darcy's law. By fixing two elements of the triplet, the third is determined using the following analytical solution for radial flow near a point source/sink in an infinite domain:

$$
\mathrm{P}_{\rho_{\mathrm{P}}}=\mathrm{P}_{\text {Well }}+\frac{|\mathrm{Q}|}{2 \pi \mathrm{K}} \ln \left(\frac{\rho_{\mathrm{P}}}{\mathrm{r}_{\text {Well }}}\right)
$$

where $\mathrm{P} \rho_{\mathrm{P}}$ is the projected pressure at radius $\rho_{\mathrm{P}}$ from the well, $\mathrm{P}_{\text {well }}$ is the pressure at the well (point measurement), and $r_{\text {well }}$ is the well radius.

Initial Conditions. The system of PDEs (1)-(3) is obviously highly nonlinear in the unknown variables $\mathrm{P}$, and $\mathrm{K}$. Consequently it is important to start with good initial guess estimates of $\mathrm{P}$ and $\mathrm{K}$ for the Newton-Raphson liberalization process used in the numerical PDE solver. The procedure consists of decoupling (1) and (3) and solving them separately. The $\mathrm{P}$ initial trial is selected based on solving equation (1) subject to outer and inner P-BCs. Similarly, the K initial trial is obtained by solving only the $\mathrm{K}$-equation with $\mathrm{K}$ specified only at the measurement locations. The outer-BCs are maintained at measured $\mathrm{K}$ for any given location or along any portion of $\partial \Omega$, null "K-flux", if any null flux BC is specified, and unspecified $\mathrm{K} \mathrm{BC}$ on the rest of $\partial \Omega$. 
Numerical Solution. Galerkin finite element method is used to simultaneously solve equations (1)-(3). The numerical formulation and solution of the problem is beyond the scope of this paper, and will be presented in a subsequent paper. The numerical results that are discussed in the next section are carried out using FlexPDE ${ }^{17}$, an advanced and highly flexible commercial PDE solver. The main kernel in FlexPDE includes a state-of-the-art, 2D and 3D Galerkin finite element methods with several options for choosing the basis functions, along with a very sophisticated grid generator, dynamic grid refinement, and graphical displays.

\section{Proof-of-concept Examples}

Due to lack of real field data, we will illustrate FIAT on synthetic simulated "field" data. Assuming a "ground-truth" reservoir K-map, one can solve (1) under stressed (injection/production) P-BCs. P and Q are then selected in a few locations and serve as "field" measurements. Then FIAT is used to recover the entire K-map based on $\{\mathrm{P}, \mathrm{Q}\}$ "field" measurements and $\mathrm{K}$ at well locations is directly taken from the K "ground-truth". FIAT is tested under different conditions of data sampling and location, and for different $\mathrm{K}$ "ground-truth" maps. As a first case, a single high-K inclusion in a low- $\mathrm{K}$ background $\mathrm{K}$-map is investigated. Case 2 is similar to case 1 introducing a smooth variation in $\mathrm{K}$. A third case based on a stochastic $\mathrm{K}$ random field is investigated.

Because the model is steady state and single phase, this investigation will determine only the spatial distribution of undersampling points (wells), which result in the detection of the target. The economic feasibility of the subsequent exploitation of the target will depend on the extent of the water encroachment in the target region, relative to the remainder of the domain, which is not simulated. The water encroachment certainly would necessitate an earlier detection than that for a domain in which the outer boundary condition is no-flow. This oversight is inconsequential however because the extent of encroachment relative to the progress of the exploitation is dependent on the permeability of the target. A relatively low-permeability domain/target yields both slow encroachment and high relative economic feasibility of wells exploiting the target. Under these conditions, a single-phase model is appropriate. A generalization of FIAT to unsteady state two-phase flow is ongoing and will be presented in subsequent papers.

Case 1. The inversion and undersampling investigations are conducted on a synthetic semi-circular reservoir of approximately 7 miles diameter, having constant pressure $(5,000 \mathrm{psi})$ on the outer boundary, and producing a singlephase liquid from a 2-stage oil field development scheme from a single platform. The idea is to simulate the development history, and assist decision makers in optimizing the location of infill or production well locations. Each development stage is assumed to be producing at steady state. The sequence of development is shown in Figure 1 and illustrates the two stages. Sampling points are comprised of wells only. The first stage, I, consists of 8 near-platform wells (inner-ring). The final stage, II, completes the development at 20 wells (outerring). The average well production is set at $6,370 \mathrm{~b} / \mathrm{d}$ and the well pressures vary from 2,000 to 3,500 psi. A high permeability region on the eastern portion of the field is also depicted in Figure 2. Over the entire field the permeability varies from 100 to $450 \mathrm{md}$. The principal goal of this investigation is to determine the number of wells and locations at which FIAT is able to identify the target region sufficiently to recommend its exploitation. The pressure field obtained by solving (1) with the "ground-truth" $\mathrm{K}$ is depicted in Figure 3.

FIAT is applied using P, K, and Q at the wells. Internal domain pressure, permeability, and flux $\mathrm{BCs}$ are calculated at fictitious radii using equation (6), and will serve as new inner domain BCs. The permeability BCs on $\partial \Omega$ are allowed to vary and are not set to any specific value. Equations (1)-(3) are simultaneously solved for P and K. Figure 4 shows the inverse $\mathrm{K}$ distribution at development Stage-I. The well permeabilities range from 100 to $114 \mathrm{md}$. FIAT inverted $\mathrm{K}$ values also vary between 100 to $114 \mathrm{md}$. Clearly, the inner-ring of eight producing wells only hints that a higher permeability region exists toward the eastern side of the field.

At the development Stage-II, 12 more production wells were added for a total of 20 wells. $\mathrm{K}$ was inverted and contoured using FIAT (Figure 5). In this run, $\mathrm{K}$ varies from 100 to $175 \mathrm{md}$ and $\mathrm{K}$ is clearly increasing to the east. The increased flux into the easternmost wells was extrapolated into the eastern portion of the field to values around $145 \mathrm{md}$, whereas in the western part of the field, the values are approximately $100 \mathrm{md}$. At the end of Stage-II, we have still not sampled nor estimated the full range of original K (100 to $450 \mathrm{md}$ ). However, the inversion of $\mathrm{K}$ displays an increasing trend to the east as demonstrated in Figure 6, which magnifies the southeast portion of Figure 5. Clearly, Figure 6 shows an increasing trend of high $\mathrm{K}$ to the southeast.

Finally, to enhance subsurface reservoir characterization, three new wells in the southeastern portion of the field were added totaling 23 wells (only one additional well, properly located, may suffice to identify the high-K lens). Inversion results are presented in Figure 7. As expected, the estimated K ranges from 100 to $400 \mathrm{md}$ thus resolving a greater portion of the K "ground-truth" distribution. Not surprisingly, one of the three new wells sampled a part of the K field at 440 md. FIAT could be used to assist decision-makers in optimizing the location of production wells in their efforts to achieve more cost-effective exploration and exploitation.

Case 2. The same domain is used except that the true $\mathrm{K}$ field has a sharp interface and a high region, again on the eastern side of the field, Figure $8 \mathrm{a}$. Values of the true $\mathrm{K}$ field range from 100 to $450 \mathrm{md}$. Figure $8 \mathrm{~b}$ depicts FIAT calculated K values. These estimates obtain the same original range because one of the 20 wells falls close to the high $\mathrm{K}$ zone and the sharp change in $\mathrm{K}$ in the western portion of the field is discernible. The abrupt change in $\mathrm{K}$ is extrapolated correctly to the north and south outside the well control.

Case 3. In this case, the true $\mathrm{k}$ field is statistically generated and ranges from 60 to almost $700 \mathrm{md}$. Most of the field is 100 to $450 \mathrm{md}$ with a high in the northern part of the field and a low $\mathrm{k}$ (< $100 \mathrm{md})$ region in the southeast and southwest, Figure $9 \mathrm{a}$. Figure $9 \mathrm{~b}$ depicts the FIAT k estimate from the 20 well data set case. The resulting contour plot in general maintains the features and trends of the true k-field; however, the high region is not discernible. The low $\mathrm{k}$ bodies are not 
well captured even though some of the wells sample close to them. This is mainly due to the discrepancy between the scale of heterogeneities (integral scale) and the mesh size. Indeed, if the size of the griddling mesh and the distance between the sampling well are larger than the heterogeneity integral scale, FIAT reproduces a spatially smoother solution than the actual permeability field. Clearly, high spatial frequency variations are not captured with a 20 well data set.

\section{Summary and Conclusions}

The approach presented here simultaneously solves the forward and inverse PDEs coupled through filtered pressure gradients. This approach is called Forward-Inverse Adaptive Technique (FIAT), which employs an implicit scheme with adaptive-grid, Galerkin, numerical techniques. Results from three synthetic test cases are presented where; $\mathrm{P}, \mathrm{K}$, and production, $\mathrm{Q}$ data exist only at producing wells. The results are preliminary but the algorithm successfully inverts $\mathrm{K}$ away from the wells. Results demonstrate attainable and reasonably accurate solutions. More importantly, these results provided enhanced insight into the consequences of data undersampling.

\section{Acknowledgments}

This work was performed under the auspices of the U.S. Department of Energy by the University of California, Lawrence Livermore National Laboratory under contract No. W-7405-Eng-48. Additional financial support from DeepLook Fluid Imaging Collaboration (a consortium of oil and service companies) to LLNL under contract No. 0878-66 is appreciated.

\section{References}

1. McLaughlin, D., and L.R. Townley, A Reassessment of the Groundwater Inverse Problem, Water Resources Research., 32(5), pp. 1131-1161, 1996.

2. Ginn TR, and Cushman, JH., Inverse Methods for Subsurface Flow - A Critical Review of Stochastic Techniques. Stochastic Hydrology and Hydraulics, 4(1), pp. 1-26, 1990.

3. Yeh, W. W-G., Review of Parameter Identification Procedures in Groundwater Hydrology: The Inverse Problem, Water Resources Research., 22, pp. 95-108, 1986.

4. Vasco, DW; and Datta-Gupta, A., Integrating multiphase production history in stochastic reservoir characterization. SPE Formation Evaluation, SEP, 12(3), pp.149-156, 1997.

5. Sun, N.-Z., Inverse Problems in Groundwater Modeling, Kluwer Acad., Norwell, MA, 1994.

6. Zhu, J., Using a Hypercube to Solve Inverse Problems in Reservoir Simulations. Applications on Advanced Architecture Computers Section, Greg Astfalk, Editor, SIAM News, March, p.1-11, 1992.

7. Chawathé, A., DeepLook: Toward the Common Goal of Reservoir Characterization Through Collaboration. OTC-10785 Paper. Offshore Technology Conference. Houston, TX, 3-6 May, 1999.

8. Nelson, R.W., In Place Measurement of Permeability in Heterogeneous Media, 2, Experimental and Computational Considerations, J. Geophys. Res., 66(8), pp. 2469-2478, 1961.

9. Nelson, R.W., Conditions for Determining Areal Permeability Distributions by Calculation, J. Soc. Petrol. Eng., 2(3), pp. 223224, 1962.

10.Emsellem Y., and G. de Marsily, An Automatic Solution for the Inverse Problem. Water Resources Research, 7(5), pp. 12641283, 1971.

11.Protter, M.H., and H.F. Weinberger, Maximum Principles in Differential Equations, Springer-Verlag, New York, NY, 1984.
12.Bracewell, R.N., The Fourier Transform and Its Applications, Second Ed., Revised, McGraw-Hill, New York, NY, 1986.

13.McGillem, C.D., and G.R. Cooper, Continuous and Discrete Signal and System Analysis, Second Ed., Holt, Rinehart, and Winston, New York, NY, 1984.

14. Oden, J.T., T. Strouboulis, and P. Devloo, Adaptive Finite Element Methods for the Analysis of Inviscid Compressible Flow: Part I. Fast Refinement/Unrefinment and Moving Mesh Methods for Unstructured Meshes, Computer Methods in Applied Mechanics and Engineering, 59, pp. 327-362, 1986.

15. Mathéron, G., Elements pour une theorie des milieux poreux, 166p., Masson \& Cie, Paris, 1967.

16.Zienkiewicz and Taylor, The Finite Element Method, Volume 1 and 2, pp. 648, McGraw-Hill, 1989.

17. Nelson, R.G., FlexPDE, www.pdesolutions.com, 2001. 


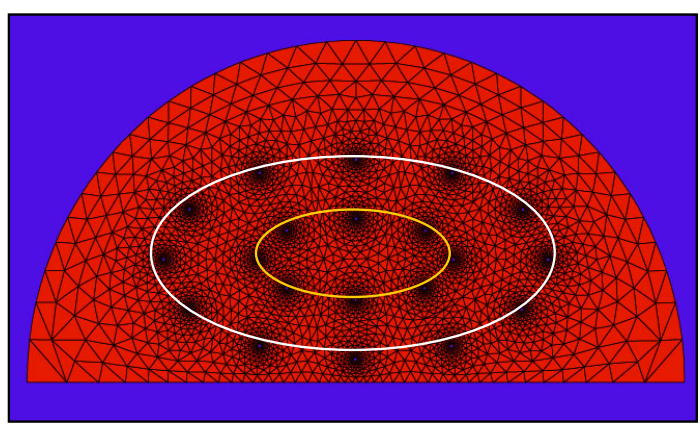

Figure 1: Sysnthetic semi-circular reservoir. Horizontal crosssection. 20 infield wells, 8 inner ring, and 12 outer-ring are depicted. Figure shows the initial finite element mesh. Higher density mesh reflects well locations.

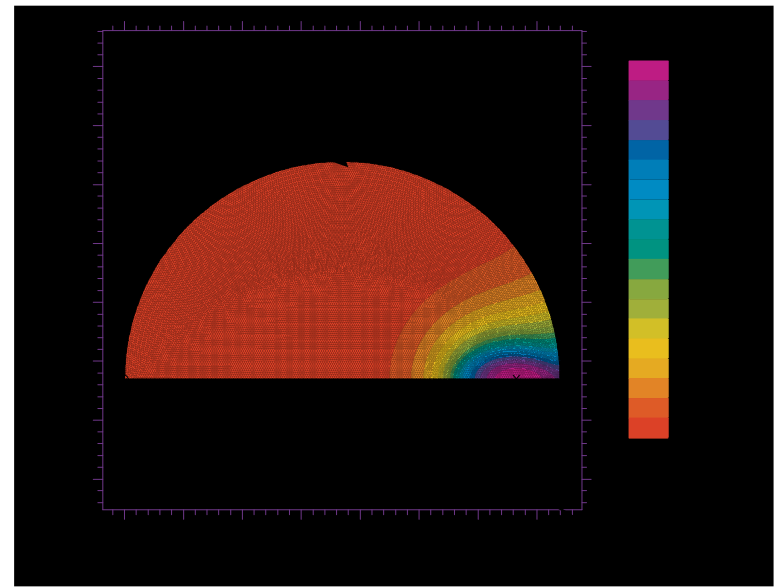

Figure 2: Ground-truth permeability field (case 1). K varies between 100 and $480 \mathrm{md}$. Pressure boundary conditions are also presented.

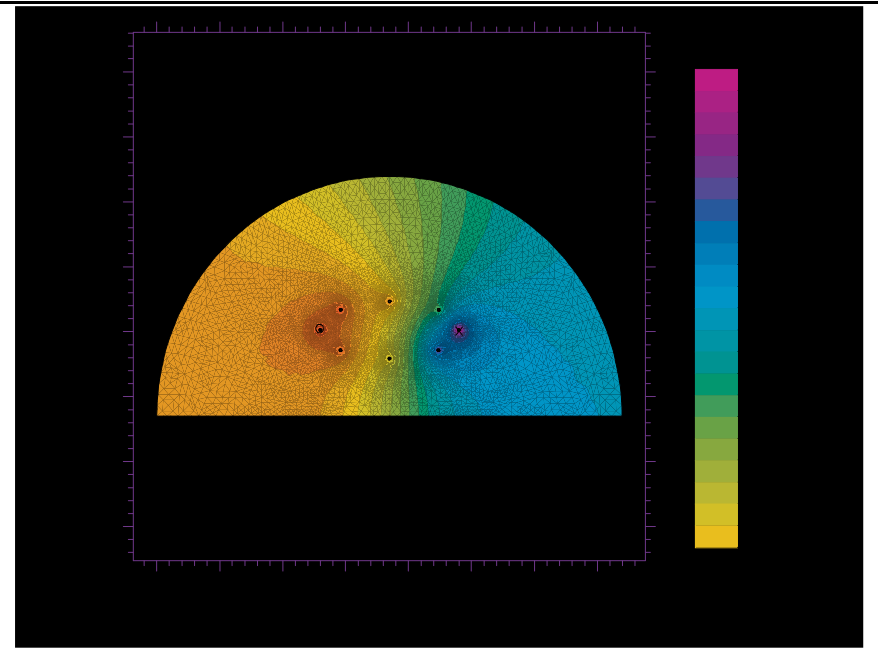

Figure 4: Stage I (8 infield wells) FIAT results. Permeability k is depicted. Permeability values vary between 103 and $114 \mathrm{md}$.

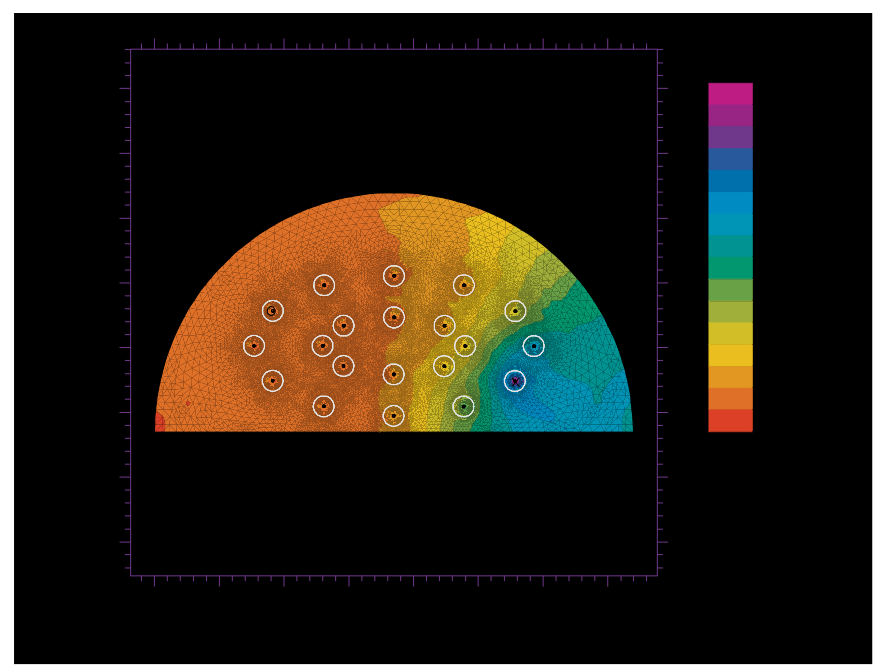

Figure 5: Stage II (20 infield wells) FIAT results. Permeability k is depicted. Permeability values vary between 95 and $175 \mathrm{md}$. White circles around the wells represent flux features $\left(\rho_{\mathrm{Q}}\right)$ to evaluate flux constraints using equation (5).

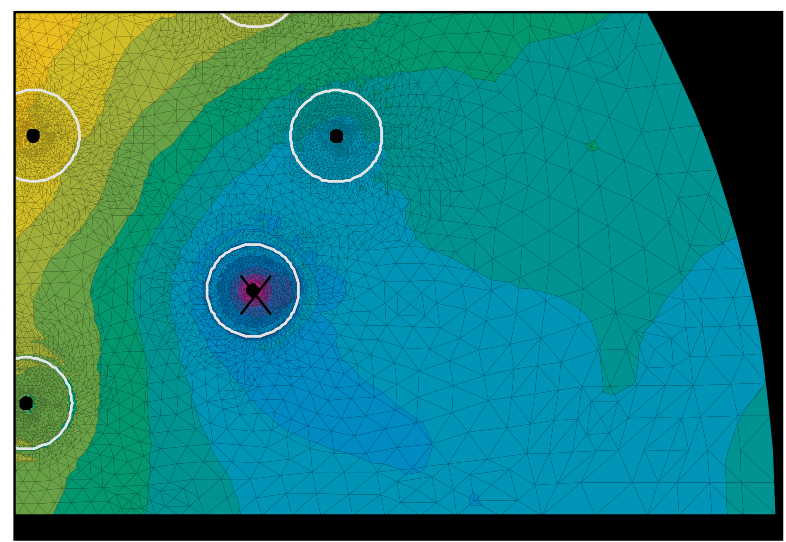

Figure 6: Zoom of the southeast portion of Figure 5. It clearly shows an increasing trend of $\mathrm{K}$ toward the southeast. More wells are needed for a final characterization stage. 


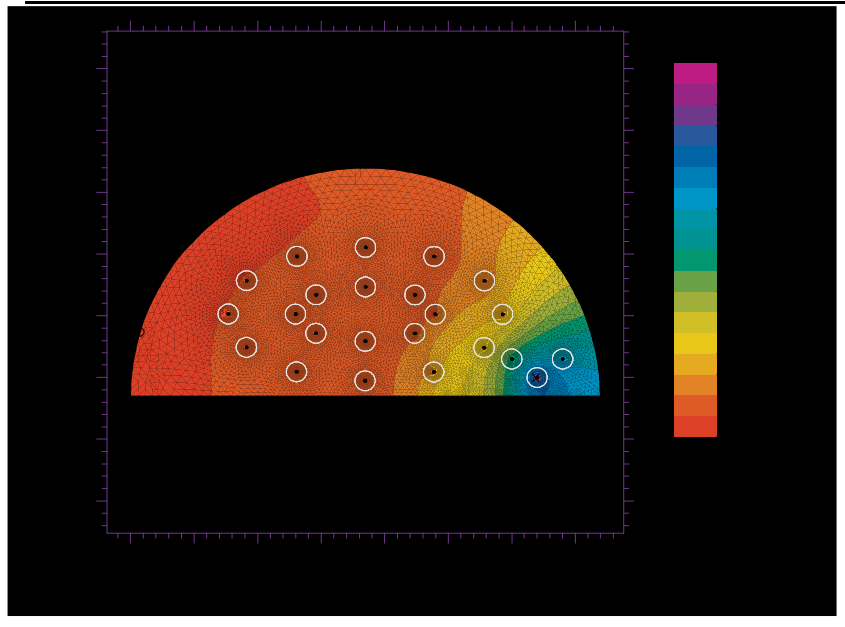

Figure 7: FIAT final stage development ( 23 wells). $\mathrm{K}$ is depicted with values varying between 90 and 440 md. Clearly FIAT recovered the high-K target within $8 \%$ error.

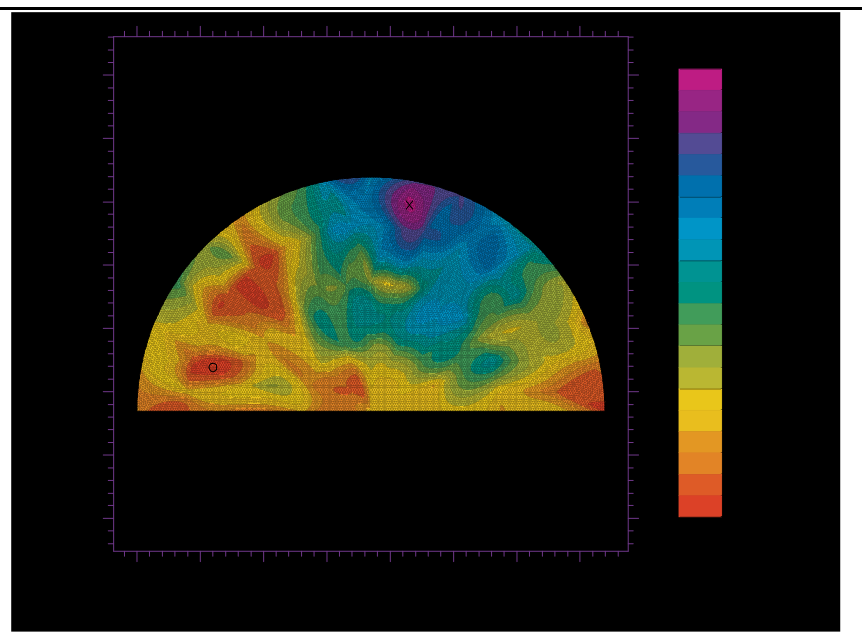

Figure 9a: Stochastic realization of a K-field.

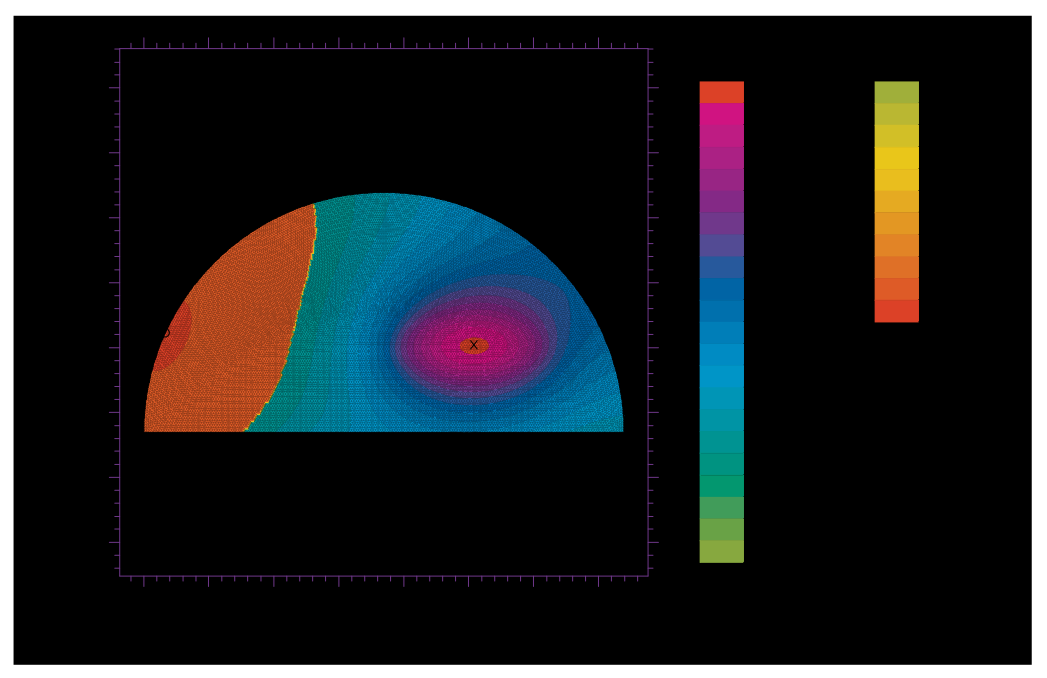

Figure 8a: Case 2. Ground-truth permeability field

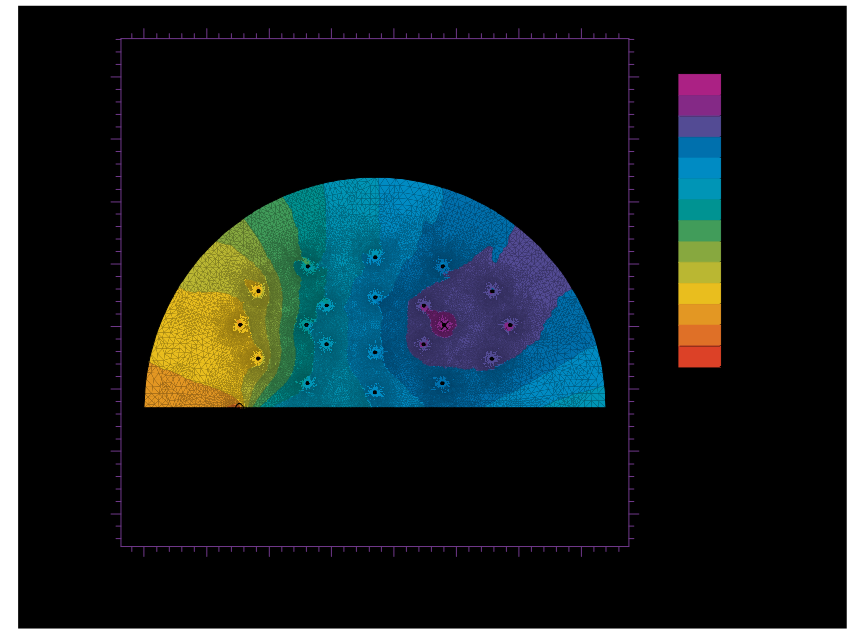

Figure 8b: Case 2. Permeability inversion. Clearly, FIAT recovered the main features of the ground-truth permeability field. The abrupt changes in $\mathrm{k}$ are discernible.

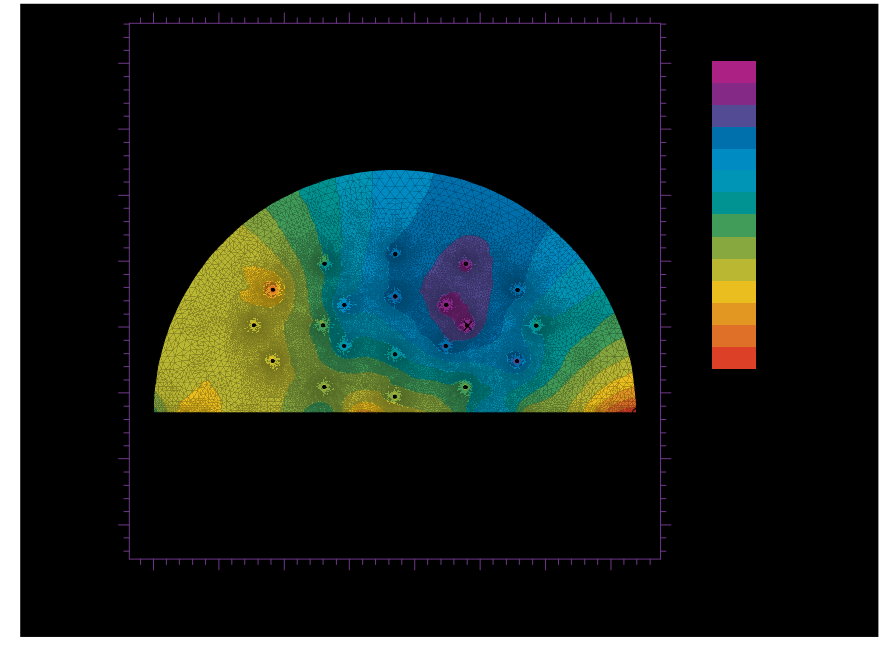

Figure 9b: K-inversion using FIAT. 